\title{
Desenho Universal para Aprendizagem e Tecnologias Digitais na Educação Matemática Inclusiva
}

\author{
Universal Design for Learning and Digital Technologies in Inclusive \\ Mathematics Education
}

\section{Diseño Universal de Aprendizaje y Tecnologías Digitales en la Educación Matemática Inclusiva}

\author{
Daiana Aparecida Stresser Fiatcoski \\ Graduanda na Universidade Federal do Paraná, Curitiba, PR, Brasil \\ E-mail: daianastresser@hotmail.com ORCID: https://orcid.org/0000-0003-0049-682X
}

\section{Anderson Roges Teixeira Góes}

Professor doutor da Universidade Federal do Paraná, Curitiba, PR, Brasil

E-mail: artgoes@ufpr.br ORCID: https://orcid.org/0000-0001-8572-3758

Recebido em 09 de setembro de 2020

Aprovado em 24 de fevereiro de 2021

Publicado em 23 de março de 2021

\section{RESUMO}

Este estudo verifica indícios do Desenho Universal para Aprendizagem (DUA) presentes no uso de Tecnologias Digitais (TDs) na Educação Matemática Inclusiva. Para tanto, são analisadas pesquisas publicadas em duas edições do Encontro Nacional de Educação Matemática: 2016 e 2019; evento que congrega maior número de pesquisadores e professores da área de Matemática no Brasil. Ao analisar os trabalhos publicados em tal evento, busca-se responder aos seguintes questionamentos: Como as TDs estão sendo utilizadas na Educação Matemática Inclusiva? É possível verificar a perspectiva do DUA ao utilizar as TDs na Educação Matemática Inclusiva? Com a metodologia adotada, de abordagem qualitativa, do tipo bibliográfica, emergem quatro temáticas: uso de tecnologias analógica e digital; uso de softwares e/ou aplicativos; uso de Ambiente Virtual de Aprendizagem; e uso de videoaula e vídeos instrutivos. Pelas análises é possível concluir que a inclusão escolar está em processo, envolvendo pesquisadores e professores no desenvolvimento de tecnologias digitais ou analógicas. Ainda, as análises revelam que é tímida a presença do DUA na Educação Matemática Inclusiva.

Palavras-chave: Tecnologias Digitais; Educação Matemática Inclusiva; Desenho Universal para Aprendizagem.

\section{ABSTRACT}

This study verifies evidence of Universal Design for Learning (UDL) present in the use of Digital Technologies (DTs) in Inclusive Mathematics Education. To this end, research published in two editions of the National Meeting on Mathematics Education is analyzed: 2016 and 2019; to answer the following questions: event that brings together the largest number of researchers and professors in the field of Mathematics in Brazil. When 
http://dx.doi.org/10.5902/1984686X55111

analyzing the works published in such an event, we seek to answer the following questions: How are DTs being used in Inclusive Mathematics Education? Is it possible to verify the perspective of UDL when using DTs in Inclusive Mathematics Education? With the adopted methodology, of qualitative approach, of bibliographic type, four themes emerge: use of analog and digital technologies; use of software and/or applications (apps); use of Virtual Learning Environment; and use of video lessons and instructional videos. Through the analysis it is possible to conclude that school inclusion is in process, involving researchers and teachers in the development of technologies digital or analog. Still, the analyzes reveal that the presence of UDL in Inclusive Mathematics Education is timid.

Keywords: Digital Technologies; Inclusive Mathematics Education; Universal Design for Learning.

\section{RESUMEN}

Este estudio verifica la evidencia del Diseño Universal de Aprendizaje (DUA) presente en el uso de las Tecnologías Digitales (TDs) en la Educación Matemática Inclusiva. Con este fin, se analiza la investigación publicada en dos ediciones de la Reunión Nacional sobre Educación Matemática: 2016 y 2019; evento que reúne al mayor número de investigadores y profesores en el campo de las Matemáticas en Brasil. Al analizar los trabajos publicados en tal evento, buscamos dar respuesta a las siguientes preguntas: ¿Cómo se usan las TDs en la Educación Matemática Inclusiva? ¿Es posible verificar la perspectiva del DUA cuando se usan TDs en Educación Matemática Inclusiva? Con la metodología adoptada, de enfoque cualitativo, de tipo bibliográfico, surgen cuatro temas: uso de tecnologías analógica y digital; uso de software y/o aplicaciones (apps); uso del Entorno Virtual de Aprendizaje; y uso de lecciones en video y videos instructivos. A través del análisis, es posible concluir que la inclusión escolar está en proceso, tenendo la participación de investigadores y docentes en el desarrollo de tecnologías, digitales o analógicas. Aún, los análisis revelan que la presencia de DUA en la Educación Matemática Inclusiva es tímida.

Palabras clave: Tecnologías Digitales; Educación Matemática Inclusiva; Diseño Universal de Aprendizaje.

\section{Introdução}

O Brasil assumiu compromissos para que as pessoas com deficiência fossem efetivamente incluídas nas escolas regulares, universalizando o acesso e promovendo a equidade, como se destaca na Constituição Federal (BRASIL, 1988), artigo 205, em que a educação é um direito de todos os cidadãos e, no artigo 208, reitera o atendimento educacional especializado à pessoa com deficiência, preferencialmente na rede regular de ensino.

Em relação ao movimento mundial de direitos das pessoas com deficiências, destaca-se a Conferência Mundial sobre Educação Especial, em 1994, na cidade 
http://dx.doi.org/10.5902/1984686X55111

Salamanca, Espanha, cujas discussões culminaram no documento conhecido como Declaração de Salamanca em que diversos governos e organizações internacionais firmaram o compromisso pela inclusão e defendem a inclusão de pessoas com deficiência em todos os níveis e modalidades de ensino.

Alinhando as práticas da educação especial com as orientações advindas da Convenção Internacional do Direito das Pessoas com Deficiência, documento aprovado em 2007 pela Organização das Nações Unidas (ONU) e que foi ratificado no Brasil em 2009, assumindo, por sua vez, teor semelhante a uma legislação, o governo brasileiro determina a Política Nacional da Educação Especial na Perspectiva Inclusiva (PNEEPI) em 2008. Tal documento apresenta histórico da educação especial, trata entre outros aspectos de princípios como igualdade e equidade enquanto valores a serem buscados, indicando os rumos das políticas nacionais. Em 2015, é promulgada a Lei Brasileira de Inclusão da Pessoa com Deficiência, lei no 13.146, assegurando um sistema educacional inclusivo em todos os níveis, garantindo o direito de que todas as pessoas com deficiência tenham acesso e permanência no ensino regular com as ferramentas educacionais necessárias para tanto.

Apesar das diversas legislações, ainda se percebe na atualidade a dificuldade no entendimento das demandas da educação inclusiva, desvirtuando o papel da educação especial que não é um tipo de ensino exclusivo, mas sim um modelo de educação de ensino comum. Maria Teresa Égler Mantoan já afirmava que

\begin{abstract}
A inclusão é uma inovação que implica um esforço de modernização e de reestruturação das condições atuais da maioria de nossas escolas (especialmente as de nível básico), ao assumirem que as dificuldades de alguns estudantes não são apenas deles, mas resultam, em grande parte, do modo como o ensino é ministrado e de como a aprendizagem é concebida e avaliada. (MANTOAN, 2003, p. 32)
\end{abstract}

Neste sentido, além da presença do estudante com necessidades educacionais especiais na rede regular de ensino deve-se ter a preocupação em incluí-lo efetivamente na rotina escolar, pois apenas o acesso não é garantia de inclusão, tendo o docente a compreensão de que existem diferentes formas de aprender e ensinar. $\mathrm{O}$ ambiente escolar deve proporcionar diferentes ferramentas pedagógicas (metodologias, artefatos e outros), aqui denominadas de tecnologias educacionais, para contribuir com a aprendizagem dos estudantes na perspectiva inclusiva. 
http://dx.doi.org/10.5902/1984686X55111

A tecnologia posta à disposição dos estudantes tem por objetivo desenvolver as possibilidades individuais, tanto cognitivas como estéticas, através das múltiplas utilizações que o docente pode realizar nos espaços de interação grupal. (LETWIN, 1997, p. 10)

O uso de tecnologias pode criar inúmeras possibilidades de trabalho em sala de aula e abordar conteúdos de forma interativa, envolvendo o "mundo" do estudante favorecendo a aprendizagem e, por sua vez, proporcionando possibilidades de inclusão aos estudantes. Desta forma, na atualidade, é inevitável que as diversas tecnologias façam parte do ecossistema da sala de aula para auxiliar os professores no processo de ensino e aprendizagem dos estudantes. Dentre as diversas tecnologias educacionais para o ensino e aprendizagem, em específico da matemática, área de discussão deste estudo, desde o lápis às atuais tecnologias, destacamos as Tecnologias Digitais (TDs) como os dispositivos móveis (smartphones, tablets e outros), softwares de geometria, lousa digital, sites de internet com conteúdo matemáticos, vídeos elaborados para estudantes ou por estudantes à disposição de seus usuários.

$\mathrm{Na}$ Educação Inclusiva é importante a perspectiva do Desenho Universal para Aprendizagem (DUA) que visa adequar estratégias e recursos de trabalho em sala de aula para a participação de todos os estudantes e não apenas iniciativas individuais, não removendo os desafios educacionais, mas removendo barreiras ao acesso dos estudantes à Educação (CAST, 2018).

Tendo essas considerações de temática a serem discutidas na próxima seção, este estudo tem o objetivo de verificar os indícios do DUA presentes no uso de Tecnologias Digitais na Educação Matemática Inclusiva, campo da Educação Matemática em que estão inseridas as pesquisas para viabilizar a apropriação do conhecimento matemático por todos os estudantes. Para isso, segundo o GT13 da Sociedade Brasileira de Educação Matemática, as pesquisas nesse campo buscam discutir as

práticas escolares e culturais, políticas educacionais, formação de professores, desempenho acadêmico e experiência com a matemática fora do contexto escolar de pessoas historicamente marginalizadas, em particular pessoas: com deficiências ou/e transtornos; com altas habilidades; com dificuldades específicas de aprendizagem de matemática; em situação de risco ou vulnerabilidade social. (SBEM, 2020, sp.)

Os questionamentos a serem respondidos neste estudo são: Como as TDs estão sendo utilizadas na Educação Matemática Inclusiva? É possível verificar a perspectiva do DUA ao utilizar as TDs na Educação Matemática Inclusiva? 
http://dx.doi.org/10.5902/1984686X55111

Diante dessas questões, esse estudo, de abordagem qualitativa, do tipo bibliográfica, analisa publicações que trazem práticas docente em matemática relatadas em duas edições do Encontro Nacional de Educação Matemática (ENEM), verificando de que forma as tecnologias são utilizadas em prol de uma Educação Inclusiva. O ENEM é um evento promovido pela Sociedade Brasileira de Educação Matemática (SBEM), sendo considerado:

O evento é o mais importante no âmbito nacional, porque congrega o universo dos segmentos envolvidos com a Educação Matemática: professores da Educação Básica, Professores e Estudantes das Licenciaturas em Matemática e em Pedagogia, Estudantes da Pós-graduação e Pesquisadores. (SBEM, 2019, sp.)

Este texto está organizado em cinco seções, sendo que a seguir são apresentados os referenciais sobre DUA e Tecnologias Educacionais.

\section{Referenciais do presente estudo}

Essa seção apresenta os referenciais relacionados ao Desenho Universal para Aprendizagem e às Tecnologias Educacionais.

\section{Desenho Universal para Aprendizagem}

O DUA teve origem no Desenho Universal (DU) surgido na década de $80 \mathrm{com}$ o arquiteto Ron Mace ao projetar prédios e espaços públicos para acesso a todos, sem nenhuma limitação. Esta concepção de planejamento acessível foi percebida como uma solução que resolve problemas em várias áreas, sendo ampliada, com denominação de DUA, para a área educacional que possui muitas barreiras pedagógicas que devem ser eliminadas para que haja um ensino efetivo de todos os estudantes. Com isso, surge a possibilidade de que todos aprendam com o mesmo recurso, removendo barreiras, contribuindo para a aprendizagem de estudantes com e sem deficiência, proporcionando a inclusão no ambiente escolar.

O DUA possui três princípios orientadores da aprendizagem que são conhecidos como redes: Rede Afetiva ou o porquê da aprendizagem; a Rede de Conhecimento ou o quê da aprendizagem; e a Rede Estratégica ou o como da aprendizagem (ZERBATO; MENDES, 2018). Essas redes são estimuladas por meio de interesses, apresentando conteúdos e diferenciando as maneiras de expressar o que os estudantes sabem. Com 
http://dx.doi.org/10.5902/1984686X55111

isso, essa perspectiva engaja e incentiva estudantes de maneira autônoma, lúdica e interativa, sendo as diversas tecnologias aliadas dos professores, pois

\begin{abstract}
Atualmente muito se discute sobre o uso das tecnologias emergentes na educação, em especial sobre a contribuição do computador no processo de ensino e aprendizagem. Compreende-se as novas tecnologias como auxiliares nesse processo, cabendo ao professor o papel da mediação para o uso significativo desses recursos. (LUZ; GÓES, 2016, p. 47)
\end{abstract}

Quando se discute tecnologia logo se faz a relação com computadores modernos, celulares cheios de funções, artefatos como óculos de realidade virtual, no entanto a tecnologia é muito ampla e contempla desde objetos simples do dia a dia até produtos sofisticados como telefones móveis, e compreende também projetos e metodologias, usos esses que se fazem presentes no cotidiano escolar.

\title{
Tecnologias Educacionais
}

A tecnologia é um "conjunto de conhecimentos e princípios científicos que se aplicam ao planejamento, à construção e à utilização de um equipamento em determinado tipo de atividade" (KENSKI, 2007, p. 24). O avanço das tecnologias mudou a maneira de realizar atividades cotidianas e com tantas mudanças é de se esperar que ela altere também a forma como se aprende e se ensina, pois, se antes os estudantes eram educados para usar a tecnologia, hoje as tecnologias servem para educar (KENSKI, 2007; LUZ; GÓES, 2016).

No ambiente escolar, compreendemos as tecnologias classificadas como: Físicas - são as inovações de instrumentais físicos, tais como: caneta
esferográfica, livro, telefone, aparelho de celular, satélites, computadores;

Organizadoras - são as formas de como nos relacionamos com o mundo e como diversos sistemas produtivos estão organizados;

Simbólicas - estão relacionadas com a forma de comunicação entre as pessoas, desde o modo como estão estruturados os idiomas escritos e falados até como as pessoas se comunicam. (SANCHO, 1998, p. 133)

O uso da tecnologia na educação possui desafios entre eles o de oportunizar a equidade entre os estudantes, fazendo com que cada estudante disfrute da melhor maneira de aprender, garantindo seu ritmo a partir dos seus interesses, conforme seu perfil de aprendizagem. Dentre as tecnologias presentes no ambiente escolar destacam-se as Tecnologias Digitais (TDs) que aliada à educação representam recursos cada vez mais diversificados, interativos e dinâmicos que ajudam o estudante a compreender e aplicar o conhecimento. A diversificação apoia o professor oportunizando a criação de novas 
http://dx.doi.org/10.5902/1984686X55111

estratégias pedagógicas, fazendo com que o conteúdo esteja cada vez mais disponível e acessível aos estudantes.

Segundo Pierre Lévy deve-se evitar o risco de digitalizar os processos educacionais, ou seja, simplesmente trocar a lousa comum pela lousa digital, livro de papel pelo livro digital ou mesmo a aula convencional pela videoaula, deve-se considerar que a conectividade precisa ser naturalmente vivida no ambiente escolar.

É nesse contexto que este estudo procura responder as questões norteadoras apresentadas na introdução deste texto. Para isso, a próxima seção apresenta a metodologia da pesquisa em que foram analisados os anais das duas últimas edições do evento ENEM (2016 e 2019).

\section{Metodologia}

A presente pesquisa possui abordagem qualitativa, do tipo bibliográfica, envolvendo trabalhos publicados em edições do Encontro Nacional de Educação Matemática (ENEM), 2016 e 2019. Para isso, a metodologia parte da seleção dos trabalhos, utilizando o primeiro critério de inclusão/exclusão que consiste em identificar trabalhos cujos títulos possuem palavras que remetem a: inclusivo(a), assistivo(a), Desenho Universal para Aprendizagem, adaptado(a) entre outras, bem como, o nome de deficiência específica como Síndrome de Down, autismo, deficiência visual e suas ramificações como cegueira e cegos, e deficiência auditiva e ramificações, TDAH e quaisquer outras palavras que se referem às pessoas com deficiência.

O segundo critério de inclusão/exclusão analisa de forma completa o título dos trabalhos selecionados com o primeiro critério de inclusão/exclusão, verificando se os mesmos indicam tratar de pessoas com deficiência.

No terceiro critério de inclusão/exclusão são analisados os resumos dos trabalhos e selecionados aqueles que indicam fazer uso das TDs no processo de ensino e aprendizagem, sejam como recursos para utilização dos estudantes, quanto recurso para o trabalho docente. Para tanto, os textos lidos integralmente possibilitaram a coleta de informações relativas a autor(es), título, instituição, objetivo, materiais utilizados, público alvo, metodologia e a conclusão a que chegaram os autores, para que seja possível a compreensão das mesmas.

Com os dados coletados foram criados grupos de análise de tal forma que cada comunicação esteja classificada em apenas um grupo. Para caracterizar cada grupo são 
http://dx.doi.org/10.5902/1984686X55111

apresentados recortes dos trabalhos e, com isso, é possível analisar como as tecnologias educacionais, sobretudo as TDs, estão sendo utilizadas na educação e na disciplina de matemática como recurso didático.

Os resultados da coleta de dados desta metodologia são apresentados na subseção a seguir.

\section{Resultados}

Como dito anteriormente, a pesquisa analisa os trabalhos publicados no ENEM, evento de maior divulgação de pesquisas e prática de Educação Matemática no Brasil. Como seleção temporal, procurando verificar os trabalhos atuais, foram analisadas duas edições do evento: XII ENEM, realizado na cidade de São Paulo/SP em 2016; e XIII ENEM, realizado na cidade de Cuiabá/MT no ano de 2019.

O XII ENEM apresenta 1434 trabalhos, classificados em 971 comunicações científicas e 463 relatos de experiência. Esses trabalhos foram submetidos ao primeiro critério de inclusão de seleção dos textos, sendo selecionados 54 trabalhos (42 comunicações científicas e 12 relatos de experiência). Quanto à edição XIII ENEM, foram divulgados 1573 trabalhos, classificados pela organização do evento em 25 eixos temáticos, sendo que no de número 5, denominado de Práticas Inclusivas em Educação Matemática, estão incluídas as comunicações cientificas, os relatos de experiência e os minicursos relacionados à Educação Matemática Inclusiva. Assim, o presente estudo realizou a análise das publicações contidas nesse eixo, sendo selecionados, no primeiro critério de inclusão, 59 trabalhos. Dessa forma, com o primeiro critério de inclusão/exclusão de trabalhos para análise, foram selecionados 113 textos (54 selecionados no XII ENEM e 59 selecionados no XIII).

Os 113 trabalhos selecionados foram submetidos ao segundo critério de inclusão/exclusão que consiste na análise dos títulos, sendo excluídos, dentre outros, trabalhos que apresentam informações indicando não abordar de pessoas com deficiências e, sim, a educação matemática na terceira idade. Com isso, permaneceram para análise dos resumos 105 trabalhos que tiveram seus resumos lidos com a finalidade de verificar se mencionam uso das TDs no processo de ensino e aprendizagem. Essa análise resultou na seleção de 14 trabalhos para a leitura na íntegra e analisados no presente estudo.

A Tabela 01 apresenta os dados quantitativos desse processo de seleção dos textos a serem analisados na leitura integral. 
http://dx.doi.org/10.5902/1984686X55111

Tabela 1 - Quantitativo de trabalhos selecionados em cada primeiro critério de inclusão/exclusão

\begin{tabular}{c|c|c|c|c}
\hline \multirow{2}{*}{ Edição } & \multirow{2}{*}{ Publicações } & \multicolumn{3}{|c}{ Critério de inclusão/exclusão } \\
& & Primeiro & Segundo & Terceiro \\
\hline XII & 1434 & 54 & 53 & 11 \\
\hline XIII & 1573 & 59 & 52 & 3 \\
\hline Total & $\mathbf{3 0 0 7}$ & $\mathbf{1 1 3}$ & $\mathbf{1 0 5}$ & $\mathbf{1 4}$ \\
\hline
\end{tabular}

Fonte: Os autores (2020).

Os 14 trabalhos lidos integralmente tiveram as informações coletadas, sendo possível classifica-los em quatro temáticas relacionadas ao uso das TDs, cujo quantitativo de trabalhos presentes em cada temática, por edição do ENEM, é apresentado na Tabela 02.

Tabela 2 - Quantitativo de trabalhos selecionados em cada primeiro critério de inclusão/exclusão

\begin{tabular}{l|c|c|c}
\hline \multirow{2}{*}{ Temática } & \multicolumn{2}{|c|}{ Edição do ENEM } & \multirow{2}{*}{ Total } \\
& XII & XIII & \\
\hline Uso de tecnologias analógica e digital & 7 & 1 & $\mathbf{8}$ \\
\hline Uso de softwares e/ou aplicativos & 1 & 2 & $\mathbf{3}$ \\
\hline Uso de Ambiente Virtual de Aprendizagem & 1 & - & $\mathbf{1}$ \\
\hline Uso de videoaula e vídeos instrutivos & 2 & - & $\mathbf{2}$ \\
\hline \multicolumn{2}{c|}{ Total } \\
\hline
\end{tabular}

Fonte: Os autores (2020).

Na sequência, expomos a síntese dos 14 trabalhos, separados por temáticas.

a) Uso de tecnologias analógica e digital

São oito pesquisas (CAMELO et al., 2016; CAMARGO et al. 2016; CIVARDI, 2016; GUIMARÃES; MATHIAS, 2016; KALEFF; ROSA; OLIVEIRA, 2016; ROCHA; SCHULÜNZEN, 2016; ZANQUETTA; NOGUEIRA, 2016; AMORIM, 2019) classificadas nesta temática, cujas tecnologias utilizadas são analógicas e digitais.

Para Camelo et al. (2016), o tato é o meio mais eficiente de aprendizagem para estudantes com deficiência visual, sendo os materiais manipulativos fundamentais no processo de aprendizagem. As autoras adaptaram atividades utilizando materiais como EVA para abordar o tema polinômios, ainda, salientam a importância do uso de ferramentas manipulativas e tecnológicas como o multiplano e dos softwares NVDA e DOSVOX na tutoria de dois estudantes cegos, um do curso de licenciatura em matemática e o outro do 
http://dx.doi.org/10.5902/1984686X55111

terceiro ano do ensino médio. Um dos pesquisados destaca que o uso do DOSVOX esteja em provas e trabalhos. As autoras concluem que

A utilização de materiais manipulativos se faz de fundamental importância, como foi possível perceber ao longo do texto, tendo em vista a necessidade de colocar em prática a criação de métodos e estratégias pedagógicas alternativas e significativas para que ocorra a aprendizagem de estudantes com deficiência visual. (CAMELO et al., 2016, p. 10)

Camargo et al. (2016) desenvolveram projeto para adaptar atividades do acervo do laboratório de matemática (LABIM) da Universidade Federal do Sergipe quando perceberam que as atividades não atendiam aos deficientes visuais e auditivos. Com isso, avaliam a acessibilidade dos materiais analógicos existentes no acervo do LABIM e a posterior adaptação necessária, bem como, a aplicação das mesmas. A TD utilizada na pesquisa é o software Braille Fácil para transcrição de textos, facilitando o entendimento dos conteúdos por parte dos deficientes visuais. Como conclusão os autores indicam que

A partir do nosso trabalho, pudemos perceber o quanto é longo o caminho a ser percorrido para tornar nossas atividades efetivamente inclusivas. Percebemos também que ao realizar as adaptações devemos ter bastante atenção aos detalhes, visando sempre diminuir as dificuldades sentidas por esses alunos. (CAMARGO et al., 2015, p. 10-11)

O estudo realizado por Civardi (2016) apresenta os resultados que demonstram sua experiência docente e investigativa no campo da Educação Matemática Inclusiva. A autora demonstra a criação de materiais pedagógicos manipuláveis adaptados na perspectiva das tecnologias assistivas e um aplicativo com jogo denominado de E-drons para atender a estudantes com baixa visão. A elaboração de diversos recursos para abordar conceitos de aritmética e geometria espacial está pautada na diversidade de estudantes que "impõe desafios sobre como propor um currículo, metodologias, recursos didáticos, estratégias, conteúdos etc. que impulsionem o aprendizado matemático de todos os que ali o estão buscando." (CIVARDI, 2016, p. 11). O aplicativo contém sequência de atividades de geometria espacial e seu desenvolvimento teve a participação de dois estudantes cegos, professores e a equipe do Centro Integrado de Aprendizagem em Rede da Universidade Federal de Goiás. Os resultados

[...] mostram que o processo de ensino-aprendizagem da matemática na escola não pode ser compreendido de forma desconexa com as investigações realizadas nas universidades. Ambas as instituições têm relevante papel no desenvolvimento cognitivo dos diferentes sujeitos, inseridos no ambiente educacional. (CIVARDI, 2016, p. 11) 
http://dx.doi.org/10.5902/1984686X55111

A pesquisa desenvolvida por Guimarães e Mathias (2016) surgiu quando um dos autores, após um estágio em uma escola para surdos, notou que havia poucos professores de matemática surdos, com isso, resolveu unir sua condição de surdez à possibilidade de ensinar outros estudantes com mesma deficiência. A metodologia da pesquisa utiliza como tecnologias imagens e conteúdos disponíveis na internet. Dentre os conceitos matemáticos de maior dificuldade de compreensão pelos estudantes foi verificado os polinômios, que foram solucionados com a utilização de recursos visuais proporcionados por papéis coloridos. Ainda, os autores indicam que na pesquisa de campo realizada junto aos professores da escola de surdos de Santa Maria - RS, foram indicadas dificuldades como a comunicação pela falta de tradução de termos do português para a linguagem de sinais. Tal observação é reforçada nas conclusões do texto:

[...] a falta de sinais específicos que possam ajudar o professor ensinar e o aluno aprender, além de professores que saibam explicar os conteúdos na língua de sinais, utilizando recursos visuais e concretos que melhoram, e muito, o entendimento do conteúdo que se pretende ensinar. (GUIMARÃES; MATHIAS, 2016, p. 7)

A pesquisa de Kaleff, Rosa e Oliveira (2016) visa a criação de materiais manipulativos de baixo custo adequados ao ensino de matemática em classes regulares ou especializadas. O público alvo são estudantes com e sem deficiência dos ensinos fundamental e médio. A ideia central do projeto é a criação de um catálogo de atividades inclusivas concretas tendo como base os recursos virtuais desenvolvidos. Esses materiais são parte do Laboratório de Ensino de Geometria (LEG) da Universidade Federal Fluminense (UFF), que desde 2008 procura incluir estudantes com deficiência na rede regular de ensino e prepara os docentes da universidade para trabalhar com esse público. Os autores destacam o uso do aplicativo DOSVOX como um instrumento necessário para a comunicação dos deficientes visuais, concluindo que

As aplicações dos experimentos educacionais apresentados têm nos mostrado que eles efetivamente auxiliam o educando vidente e também aquele com deficiência visual na construção de seu conhecimento e no desenvolvimento da habilidade da visualização de determinados conceitos geométricos. (KALEFF; ROSA; OLIVEIRA, 2016, p. 11)

Rocha e Schlünzen (2016) apresentam pesquisa sobre tema gestão ambiental, cujos participantes são estudantes do $6^{\circ}$ ano de uma escola estadual, no município de Presidente Prudente - SP. São discutidas a abordagem Construcionista, Contextualizada e Significativa (CSS) e a metodologia de projetos constituídas em três etapas: temas para pesquisar na internet; pesquisa de campo para visualizar como é a gestão do lixo da escola; 
http://dx.doi.org/10.5902/1984686X55111

elaboração, sistematização dos resultados e criação de um blog. Dentre os participantes da pesquisa há dois estudantes de inclusão com deficiência intelectual que tiveram reforço das atividades na sala de recursos multifuncional. $O$ trabalho não estava concluído até o momento da publicação, mas como resultados parciais os autores indicam que

[...] não se pode dizer que com esse projeto houve mudanças de conduta, tanto na postura dos estudantes quanto nas práticas das professoras, no entanto, pode se refletir que esse foi um passo de investigação que tem possibilitado apresentar fragilidades existentes na escola, mas, ao mesmo tempo, potencialidades de que a mudança é possível. (ROCHA; SCHULÜNZEN, 2016, p. 11)

Zanqueta e Nogueira (2016) descrevem uma sequência didática com dois estudantes do $6^{\circ}$ ano do ensino fundamental, diagnosticados TDAH e surdos, além de discussões sobre duas visões principais do TDAH (organicista e a visão histórica Cultural e sua relação com a sociedade). A sequência didática possui 32 atividades, distribuídas em dois blocos, que contemplam o sistema de números decimais e campo aditivo. As atividades são adaptações e criações, com tradução em LIBRAS e outras tecnologias além da digital, como materiais manipuláveis (fichas sobrepostas, jogo do supertrunfo, quadro valor de lugar, material dourado e jogo do prato de papelão). As autoras destacaram que a dinâmica

[...] favoreceu a troca de ideias e o desenvolvimento da autonomia, proporcionando um avanço qualitativo do raciocínio; aumentou a coragem em enfrentar desafios e criar novos processos de cálculos (novo pelo menos para o estudante); aumentou a capacidade de concentração dos estudantes nas aulas; concorreu para a compreensão do conceito e dos diferentes significados do número; favoreceu o domínio de números de ordens elevadas; colaborou para a compreensão e 0 enriquecimento e a flexibilização dos procedimentos algorítmicos. (ZANQUETTA; NOGUEIRA, 2016, p. 10)

Amorim (2019) relata a experiência de adaptar um álbum de figurinhas da Copa do Mundo para um estudante cego quando soube que tal estudante era apaixonado por figurinhas e pela seleção brasileira. No primeiro momento, empenhou-se em conseguir figurinhas adaptadas, mas não teve sucesso. Com isso utilizou os recursos tecnológicos disponíveis em seu local de trabalho, como o software Braille Fácil para transformar texto em Braile. Assim, com uma cela Braille transferiu o texto para o papel e desenhou retângulos, numerando-os nos locais em que o usuário do álbum deveria colar a figura. $\mathrm{O}$ sucesso do álbum foi grande e, até o momento da publicação, o autor já havia elaborado mais de 100 cópias do projeto para estudantes, professores e colegas de trabalho. Após a confecção do álbum o autor percebeu que o material poderia ser um rico recurso didático para utilização em atividades matemáticas divertidas, incentivando estudantes em 
http://dx.doi.org/10.5902/1984686X55111

conteúdos como aritmética, geometria, construção de tabelas e gráficos ou mesmo na leitura Braille. Em suas considerações finais, o autor indica que "o álbum de figurinhas adaptado pode oferecer possibilidades ao ensino de Matemática para alunos cegos por meio de atividades que explorem e desenvolvam o conhecimento matemático nos estudantes." (AMORIM, 2019, p. 7), ainda, finaliza o texto com o desejo de que

[...] iniciativas como esta sejam motivo de inspiração a professores de alunos com deficiência visual que se dedicam e buscam oferecer um ensino de qualidade para esses estudantes nos ambientes escolares nos quais estão inseridos. (AMORIM, 2019, p. 8)

b) Uso de softwares e/ou aplicativos

As pesquisas classificadas nesta temática, num total de três (FRANZIN; ZWAN; ROSISKI, 2016; MORAIS; SILVA; FERNANDES, 2019; SANTOS; FERNANDES, 2019), utilizam em suas pesquisas softwares e/ou aplicativos.

O objetivo da pesquisa de Franzin, Zwan e Rosiski (2016) é a elaboração e organização materiais didáticos de aprendizagem para estudantes surdos com a participação de bolsistas do ensino médio, sendo um deles, um estudante surdo. As autoras apresentam os marcos legais e avanços na inclusão escolar e afirmam que as pessoas com deficiência auditiva possuem sua própria cultura e linguagem característica para sua comunicação, LIBRAS. São utilizadas diversas tecnologias digitais na pesquisa como internet, softwares matemáticos, aplicativos para celulares, jogos envolvendo os conteúdos trabalhados, câmeras fotográficas, filmadoras e, principalmente, o uso da lousa digital como meio de inclusão do estudante nas aulas. Algumas adaptações de materiais também foram realizadas, utilizando conteúdos do ensino médio, como exemplo o teorema de Pitágoras e trigonometria. Também foram elaborados vídeos com tradução em LIBRAS e software como o Geogebra e planilhas eletrônicas. As autoras relataram que

Os resultados, a avaliação e a discussão sobre os materiais construídos, ainda estão em processo de finalização. Já possuímos alguns resultados referente a aplicabilidade do material levantado durante o transcorrer do projeto, que é avaliação do bolsista surdo relatando sua aprendizagem dos conteúdos explicados a ele por meio de materiais didáticos adaptados, usando-se de recursos tecnológicos, como a lousa digital. (FRANZIN; ZWAN; ROSISKI, 2016, p. 9)

Morais, Silva e Fernandes (2019) investigam a possibilidade do software Scracth ser utilizado no ensino de matemática em vários níveis de ensino e como torná-lo inclusivo por meio de modelagens guiadas por usuários. O projeto faz parte de um estudo simulativo, desenvolvido pelo grupo de pesquisa Rumo à Educação Matemática, criado em 2002, na 
http://dx.doi.org/10.5902/1984686X55111

Universidade Bandeirantes de São Paulo, cujo objetivo é melhorar a didática de professores que possuem estudantes de inclusão sob sua responsabilidade educacional. Além do Scracth, o grupo analisa outros softwares para ensino de matemática como o MathSticks, a Calculadora musical, o Ritmática e a Transtartaruga. As autoras discorrem sobre o termo "eficientemente diferente" como forma de remeter à pessoa com deficiência. Indicam o "uso do software Scratch no processo de ensino e de aprendizagem matemática para diversos segmentos de ensino, desde que sejam consideradas as especificidades e necessidades de cada grupo de alunos eficientemente diferentes." (MORAIS; SILVA; FERNANDES, 2019, p. 14), também apontam

[...] contribuições da linguagem de programação mediada pelo Scratch no que tange a flexibilidade do pensamento matemático e ao desenvolvimento de habilidades essenciais a formação integral do aluno como criatividade, iniciativa, curiosidade, motivação, autonomia, observação de regularidades, estímulo ao trabalho em grupo, colaborativo, valorização da diversidade, dentre outros. (MORAIS; SILVA; FERNANDES, 2019, p. 14)

Santos e Fernandes (2019) apresentam pesquisa desenvolvida na disciplina "Educação Matemática e Inclusão" do Programa de Pós-graduação em Educação da Universidade Anhanguera. Os autores abordam os Cenários Inclusivos para Aprendizagem (CIA) que "são espaços não necessariamente físicos constituídos por tarefas específica, por ferramentas mediadoras (materiais, tecnológicas e/ou semióticas) a serem empregadas na execução das tarefas, e por interações entre os diferentes atores" (SANTOS; FERNANDES, 2019, p. 3), esclarecem ainda que esses espaços possuem os princípios do Desenho Universal para Aprendizagem. Na pesquisa, são utilizados diversos aplicativos em que os estudantes podem produzir livros, mapas mentais, vídeos e jogos, não sendo objetivo a adaptação dos recursos, mas sim a criação de possibilidades para que todos os alunos possam participar das atividades. O CIA escolhido foi apresentado a uma turma do $9^{\circ}$ ano do ensino fundamental que possui estudantes com Síndrome de Down, autismo e TDAH, tornando, assim, a atividade desafiadora para os autores que tiveram que pensar em ferramentas que melhor se adequassem a este público. Quanto ao uso das tecnologias digitais apresentadas, os autores indicam que

[...] embora seja reconhecido o potencial que as tecnologias apresentam no processo de aprendizagem de alunos com algum tipo de limitação, entendemos que ainda são grandes os desafios de professores e da sociedade em geral em tornar a escola e as aulas inclusivas. Mas, sabemos, também, que se faz necessária a participação e envolvimento de todos os agentes do processo educacional com dedicação e comprometimento diário. (SANTOS; FERNANDES, 2019, p. 14) 
http://dx.doi.org/10.5902/1984686X55111

c) Uso de Ambiente Virtual de Aprendizagem

A pesquisa classificada nesta temática foi realizada por Santos e Fernandes (2016), em que os autores indicam os resultados obtidos com um curso à distância em uma proposta inclusiva, oportunizando a participação de pessoas com e sem deficiência. Para atingir tal propósito foi utilizada a perspectiva do DUA para a discussão da Educação Financeira, em que "contaram com a participação de três cegos [...], oito surdos [...] e sete pessoas que declararam não possuir deficiências" (SANTOS; FERNANDES, 2016; p. 11). A plataforma utilizada para o desenvolvimento do curso foi o Moodle, escolhida para que "[...] pudessem proporcionar aos participantes momentos de reflexão, discussão, bem como a possibilidade de formular questões que contribuíssem com a busca de possíveis soluções para os problemas apresentados." (SANTOS; FERNANDES, 2016, p. 8). Os autores ofertaram dois cursos e concluíram que o AVA é "[...] de fato acessível, que permitiu a interação entre os participantes que muitas vezes, durante as interações, já não se lembravam da especificidade do seu interlocutor." (SANTOS; FERNANDES, 2016, p. 11).

d) Uso de videoaula e vídeos instrutivos

Duas pesquisas estão classificadas nesta temática pelo fato de utilizarem as seguintes TDs: videoaula (MORAES; DUTRA, 2016) e vídeos instrutivos (ASSIS, 2016).

A pesquisa, que visa à produção de 140 vídeos com tradução em LIBRAS, foi realizada por Moraes e Dutra (2016), cujo objetivo foi facilitar o entendimento sobre conceitos matemáticos. Os participantes da pesquisa são estudantes de uma escola pública da cidade de Rio Verde - GO. Os autores concluem que

[...] os propósitos do projeto têm sido cumpridos, auxiliando no aprendizado da Matemática, gerando em seu público alvo dúvidas que induzem ao raciocínio lógico e o desejo de buscar a origem dos conceitos apresentados e descontruindo o estudar através de pura memorização. (MORAES; DUTRA, 2016, p. 8)

Ainda, destacam que as análises realizadas

[...] apontam "algumas dificuldades e tendência de erros e atrapalhes de alunos. Essas dificuldades se dão principalmente na não apropriação e não significação do conhecimento estudado. Acredita-se que estas faltas serão corrigidas com a busca da aceitação da naturalidade do conteúdo e que esta naturalidade será alcançada nas respostas dos porquês. (MORAES; DUTRA, 2016, p. 8)

Os autores também indicam que a produção dos vídeos deve ser analisada quanto sua eficácia e a viabilidade de sua utilização como recurso didático. 
http://dx.doi.org/10.5902/1984686X55111

Já a pesquisa que utiliza vídeos instrutivos foi desenvolvida por Assis (2016), onde os participantes são estudantes surdos, usuários de LIBRAS como principal forma de comunicação. Quantos aos conceitos matemáticos explorados estão a "representação de temas abstratos, tais como: figuras geométricas, funções algébricas, relações aritméticas e etc." (ASSIS, 2016, p. 1), com o objetivo de "a significação, assumida ou não, pelo sujeito surdo nas diversas formas de representação matemática" (ASSIS, 2016, p. 2), especificamente no uso da Língua Portuguesa escrita, bem como, suas influências sobre os resultados observados. O autor apresenta vídeos aos participantes para que, posteriormente, comuniquem-se de tal forma que um participante transmita ao outro o que estava vendo, sem o uso de sinais conhecidos por ambos. O autor conclui que há

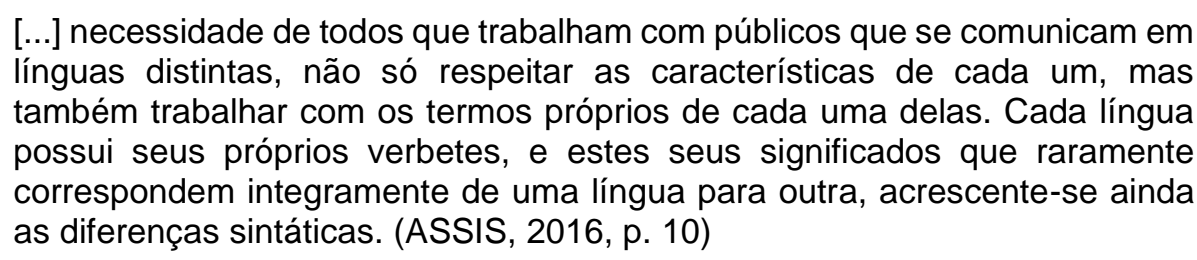

Assis (2016, p. 10) também destaca que "o fato de uma língua possuir uma quantidade menor de verbetes distintos para um determinado assunto, em nada pode representar que esta forma de comunicação é menos apta para este tema, e muito menos ainda com a capacidade cognitiva de seus usuários". Por fim, destaca "a necessidade de capacitação dos intérpretes nos termos específicos da língua alvo, bem como na Educação Matemática, em especial dos termos, representações e seus significados adotados neste campo do conhecimento." (ASSIS, 2016, p.11).

\section{O que nos revelam as pesquisas?}

A análise das 14 pesquisas selecionadas busca responder as questões que norteiam este estudo: Como as TDs estão sendo utilizadas na Educação Matemática Inclusiva? É possível verificar a perspectiva do DUA ao utilizar as TDs na Educação Matemática Inclusiva?

Pode-se observar que a maioria das pesquisas abordam a adaptação ou desenvolvimento de recursos educacionais (materiais didáticos ou metodologias) para o ensino e aprendizagem de estudantes cegos, seguido dos estudantes surdos, talvez pelo fato de considerar que esses estudantes compõem o grupo de pessoas com deficiência 
http://dx.doi.org/10.5902/1984686X55111

com maiores dificuldades, pois o mundo é repleto de informações pictóricas (LUZ; GÓES, 2016).

Por trabalharem com estudantes cegos, Camelo et al. (2016), Camargo et al. (2016) e Kaleff, Rosa e Oliveira (2016) utilizam tecnologias analógicas (materiais manipulativos) como recursos no processo de compreensão dos conceitos matemáticos. As TDs são utilizadas pelos autores como instrumentos de registro pelo estudante ou como ferramentas para transcrição das palavras em Braille.

Como forma de tornar o Moodle acessível aos participantes cegos, Santos e Fernandes (2016) instigam os demais participantes a gravarem vídeos sobre as discussões realizadas no fórum dentro do Ambiente Virtual de Aprendizagem.

Em relação aos trabalhos direcionados a estudantes surdos ou outras deficiências que não sejam a cegueira, as TDs possuem forte presença com vídeos, variados softwares de função educativa e aplicativos de dispositivos móveis.

Sobre o DUA, tal denominação é mencionada nas pesquisas de Santos e Fernandes $(2016,2019)$. Na pesquisa apresentada no XII ENEM (edição de 2016), além da experiência comentada anteriormente sobre os participantes gravarem áudio para tornar acessível a discussão com os participantes cegos, os autores instigam os participantes a realizarem vídeo com tradução em LIBRAS para incluir os participantes surdos. Já na pesquisa apresentada no XIII ENEM (edição de 2019) os autores procuram desenvolver materiais para atender estudantes com Síndrome de Down, autismo e TDAH.

Apesar de não mencionarem o DUA, há evidências dessa perspectiva na adaptação dos recursos educacionais realizada por Camelo et al. (2016), Camargo (2016) e Kaleff, Rosa e Oliveira (2016). Os materiais manipulativos pensados para estudantes que possuem deficiência visual podem ser utilizados por aqueles que não possuem tal deficiência, seja pela forma do material ou pela inclusão da simbologia Braille, permitindo que os estudantes videntes utilizem o mesmo recurso que os cegos.

Ao abordarem a metodologia de projetos com a utilização de diversos recursos educacionais, Rocha e Schlünzen (2016) trazem indícios do DUA no que se refere a forma de participação de todos os estudantes no processo de ensino e aprendizagem, independentemente de sua deficiência, que no caso da referida pesquisa são deficientes intelectuais. Também, indícios sobre o DUA são verificados na pesquisa de Moraes e Dutra (2016) ao realizarem as traduções em LIBRAS, atingindo todos os estudantes, videntes ou cegos, surdos ou ouvintes. 
http://dx.doi.org/10.5902/1984686X55111

Guimarães e Mathias (2016) e Franzin, Zwan e Rosiski (2016) não abordam o DUA, visto que o material visual é direcionado aos estudantes surdos, não sendo possível utilizar, da forma como está concebido, por estudantes cegos. O mesmo ocorre na pesquisa de Amorim (2019), apesar do material desenvolvido cumprir sua função para o aprendizado do estudante cego não há a preocupação com o estudante vidente que utilizaria a versão comercial do material. Neste mesmo sentido, Civardi (2016) faz uso da tecnologia assistiva em sua pesquisa, que são pensadas para pessoas com deficiências, mas não necessariamente atende a todos. $O$ desenvolvimento de materiais nestas concepções, de atender especificamente o estudante com deficiência, possui sua validade quanto à aprendizagem do indivíduo, no entanto, sua utilização em sala de aula pode gerar a segregação, visto que os estudantes com deficiência podem se isolar dos demais por terem materiais didáticos específicos para seu uso.

Morais, Silva e Fernandes (2019) comentam que os participantes de sua pesquisa, professores e gestores, questionam se o software Scratch pode atender a todos os estudantes: "O Scratch fala? Pode atender alunos cegos ou Síndrome de Down?" (MORAIS; SILVA; FERNANDES, 2019, p. 10). As autoras complementam que as discussões levaram "a acreditar que o segmento mais indicado para se utilizar o software era o Ensino Fundamental II até o 7ํan" (MORAIS; SILVA; FERNANDES, 2019, p. 10), mas não apresentam uma forma de utilização, demonstrando que o software pode ser utilizado na perspectiva do DUA. Neste mesmo viés, Zanqueta e Nogueira (2016) abordam um estudo de caso com dois estudantes surdos que possuem TDH, sem que os materiais utilizados pelas autoras possuam indícios da perspectiva do DUA.

Guimarães e Mathias (2016) relatam as dificuldades de comunicação pela falta de tradução de termos do português para a LIBRAS. Tal observação é objeto de estudo de outros pesquisadores na área da Educação Matemática Inclusiva, como Correa, Góes e Góes (2018) que, ao fim da pesquisa, são surpreendidos com os resultados, pois a hipótese de que a Matemática seria a área com maior ausência de sinais para tradução em LIBRAS é refutada pelos Tradutores e Intérprete de LIBRAS, indicando as áreas de Biologia, Química, Física e Língua Inglesa. Outra observação presenciada por Correa, Góes e Góes (2018) também já se anunciava nas considerações realizadas por Assis (2016), sobre a necessidade de capacitação dos TILs em termos específicos nas diversas áreas. Correa, Góes e Góes (2018) vão além, indicando que a não formação em área do conhecimento específica contribui para a dificuldade das representações dos termos. Tal consideração 
http://dx.doi.org/10.5902/1984686X55111

pode ser uma utopia vislumbrada por esses autores, mas contribuiria com o caminho que está sendo trilhado na Educação Básica para que o estudante surdo seja incluso no processo de ensino e aprendizagem.

\section{Retomadas de afirmações}

Ao analisar os trabalhos do Encontro Nacional de Educação Matemática (ENEM), evento nacional que congrega a maior quantidade de pesquisadores em Educação Matemática, bem como, professores que ensinam matemática (SBEM, 2019) foi possível observar que as TDs são utilizadas no ambiente escolar como recurso para o trabalho docente e como meio para proporcionar o ensino e aprendizagem de pessoas com deficiência.

Mesmo com as indicações de documentos oficiais que datam a década de 1990, percebe-se que as pesquisas relacionadas à inclusão escolar ainda estão trilhando seu caminho. Do total de 3007 pesquisas das duas edições do ENEM analisadas, 113 pesquisas indicam tratar de inclusão (Quadro 01), destas, 105 tratam sobre a inclusão de pessoas com deficiências. No entanto, para cumprir o objetivo deste estudo, verificar indícios do DUA presentes no uso de TDs na Educação Matemática Inclusiva, foram selecionadas 14 pesquisas na metodologia descrita.

$\mathrm{Na}$ análise realizada, quando comparada com os documentos oficiais sobre inclusão escolar, percebe-se que os autores possuem o cuidado de preservar a acessibilidade das atividades aos estudantes/participantes com deficiência, no entanto, poucas pesquisas explicitam a preocupação com os demais estudantes/participantes, o que demonstra princípios do DUA ao sugerir que os recursos sejam acessíveis a totalidade de usuários e não que sejam específicos a um receptor.

Há trabalhos que indicam a evidência da perspectiva do DUA, sem tal denominação, como pôde ser verificado em Camargo et al. (2015) e Moraes e Dutra (2016) quando há a adaptação das atividades para atender deficientes visuais e auditivos. Os recursos apresentados atendem diversos estudantes no ambiente escolar, abarcando recursos e metodologias em práticas pedagógicas inclusivas. Quando pensadas na adequação para a dificuldade de um estudante em específico é possível e remover barreiras e contribuir para a aprendizagem de todos os estudantes.

Fato positivo nas pesquisas analisadas é que não se preocupam em realizar adaptações curriculares que comumente são propostas por diversos sistemas de ensino 
http://dx.doi.org/10.5902/1984686X55111

para o trabalho com o estudante inclusivo. Mantoan (2020) afirma que ao propor um ensino individualizado ou flexibilizado para determinado estudante em função de sua deficiência, caracteriza-se uma prática discriminatória, uma vez que diminuindo o objetivo de uma atividade o professor está pressupondo que o estudante tem uma capacidade limitada para apresentar um resultado. Sobre isso, Lanutti (2020, sp.) ressalta que uma ação "rica em oportunidades, para que cada um aprenda a partir da sua capacidade, ela não precisa ser flexibilizada nem adaptada, a origem dessas atividades já contempla as diferentes necessidades e interesses dos estudantes".

Outro fato positivo é que as pesquisas não apresentam concepções errôneas, não confundem a efetiva inclusão do estudante na escola com a integração escolar. Quando um estudante está matriculado no "ensino regular" e o professor faz adaptações necessárias para que esse estudante acompanhe o conteúdo curricular, ocorre a integração desse estudante (MANTOAN, 2020), por sua vez, quando o professor repensa sua prática pedagógica e entende que toda a turma aprende de forma diferente e trabalha pensando no coletivo, não na dificuldade do indivíduo, acontece a inclusão escolar, pois este estudante vai aprender em iguais condições e segundo sua capacidade (LANUTTI, 2020).

A diversidade de tecnologias (analógicas e/ou digitais) é outro ponto positivo, corroborando com as afirmações de Letwin (1997) e Luz e Góes (2016) sobre os diversos usos das tecnologias nos espaços de interação. Isto demonstra que as pesquisas procuram formas de não diferenciar os recursos e as metodologias para os estudantes que possuem deficiência, buscando a efetivação da inclusão escolar, pois um recurso adaptado pode ser utilizado por todos os estudantes. Ainda, percebe-se o cuidado de não "digitalizar" os processos educacionais, algo que Lévy (1999) chama atenção, tanto que a maioria das pesquisas analisadas utilizam de tecnologias analógicas como recurso, ou seja, não se preocupam em "modernizar" a Educação, mas sim fazer com que os estudantes aprendam os conceitos matemáticos. Cabe ressaltar que não é uma crítica quanto ao uso das TDs, mas observações advindas de Luz e Góes (2016) sobre a necessidade de os professores verificarem se esses recursos são realmente necessários para o seu objetivo, pois cada tecnologia possui sua potencialidade que deve ser explorada em todas as suas potencialidades. Como exemplo, para o estudante cego as tecnologias digitais, que são repletas de imagens, não são úteis se não vierem acompanhadas de áudio descrição ou transcrição em Braille. Para esses estudantes, as tecnologias que proporcionem a compreensão do conhecimento pelo tato são melhor empregadas. Assim, é necessário que 
http://dx.doi.org/10.5902/1984686X55111

o professor crie estratégias pedagógicas que amparem suas aulas, uma vez que cada indivíduo tem seu ritmo e modo de aprender e é esse entrelace que pode garantir a qualidade e efetividade da educação na era tecnológica (LÉVY, 1999).

Desta forma, com este estudo é possível concluir que a inclusão ainda é um processo moroso no ambiente escolar demandando preocupação por parte dos pesquisadores e professores que estão em busca do desenvolvimento de tecnologias (recursos pedagógicos e metodologias) para atender os estudantes com deficiência inseridos nas escolas e não efetivamente incluídos. Ainda, verifica-se que a perspectiva do DUA está sendo utilizada Educação Matemática Inclusiva de forma tímida, sendo este trabalho uma contribuição para as discussões realizadas pelos pesquisadores desse campo de estudos da Educação Matemática.

\section{Referências}

AMORIM, Luigi Amato Bragança. Copa, álbum de figurinhas e matemática: Um trio inclusivo In: Encontro Nacional de Educação Matemática, anais do XIII ENEM, Cuiabá, 2019. Disponível em

https://www.sbemmatogrosso.com.br/eventos/index.php/enem/2019/paper/view/2056/11 22. Acesso em 3 de jan. 2020.

ASSIS, Claudio. A Língua Escrita e a Cognição Matemática dos Surdos. In: Encontro Nacional de Educação Matemática, anais do XII ENEM, São Paulo, 2016. Disponível em: http://www.sbembrasil.org.br/enem2016/anais/pdf/6450_2567_ID.pdf. Acesso em: 3 de jan. 2020.

BRASIL, Ministério da Educação. Política nacional de educação especial na perspectiva da educação inclusiva. Brasília: MEC/SECADI, 2008.

BRASIL. [Constituição (1988)]. Constituição da República Federativa do Brasil: promulgada em 5 de outubro de 1988.

BRASIL. Presidência da República. Decreto no 6.949, de 25 de agosto de 2009.

Promulga a Convenção Internacional sobre os Direitos das Pessoas com Deficiência e seu Protocolo Facultativo, assinados em Nova York, em 30 de março de 2007. Brasília, 2009. Disponível em: http://www.planalto.gov.br/ccivil_03/_ato2007-

2010/2009/decreto/d6949.htm. Acesso em 03 de jan. de 2020.

BRASIL. Presidência da República. Lei no 13.146, de 6 de julho de 2015. Institui a lei brasileira de inclusão da pessoa com deficiência (estatuto da pessoa com deficiência). Brasília, 2015. Disponível em: http://www.planalto.gov.br/ccivil_03/_ato20152018/2015/lei/l13146.htm. Acesso em: 11 de mar. 2020. 
CAMARGO, Erica Daiane Ferreira; ATTIE, João Paulo; OLIVEIRA, Juliene Santos; SANTANA, Rosilene Soares. $O$ enfoque da acessibilidade na produção de materiais didáticos para o ensino de matemática. In: Encontro Nacional de Educação Matemática, anais do XII ENEM, São Paulo, 2016. Disponível em: http://www.sbembrasil.org.br/enem2016/anais/pdf/5823_3516_ID.pdf. Acesso em: 3 de jan. 2020.

CAMELO, Frankisilane Gonçalves; SILVA, Maria de Fátima Dias da Silva; OLIVEIRA, Camila Tenório Freitas de; OLIVEIRA, Silvânia Cordeiro de. Experiências de ensino junto a um estudante cego: da tutoria à sua prática docente. In: Encontro Nacional de Educação Matemática, anais do XII ENEM, São Paulo, 2016. Disponível em: http://www.sbem.com.br/enem2016/anais/pdf/4614_3728_ID.pdf. Acesso em: 8 de jan. 2020.

\section{CAST - Center for Applied Special Technology. Universal Design for Learning}

Guidelines. Versão 2.2, 2018. Disponível em http://udlguidelines.cast.org. Acesso em 03 de jan. de 2020 .

CIVARDI, Jaqueline Araújo. Fazeres pedagógicos e investigativos no campo da Educação Matemática Inclusiva. In: Encontro Nacional de Educação Matemática, anais do XII ENEM, São Paulo, 2016. Disponível em:

http://www.sbem.com.br/enem2016/anais/pdf/7019_2829_ID.pdf. Acesso em: 8 de jan. 2020.

CORREA, Vanessa de Paula; GÓES, Anderson Roges Teixeira; GÓES, Heliza Colaço. Desafios enfrentados por Tradutores e Intérpretes de LIBRAS nas aulas de Matemática. Revista Educação Especial, v. 31, n. 61, abr./jun. 2018.

FRANZIN, Rozelaine de Fátima; ZWAN, Licinara Daiane; ROSINSKI. Ana Maria. A educação de surdos e o contexto tecnológico: Uma experiência com a lousa digital. In: Encontro Nacional de Educação Matemática, anais do XII ENEM, São Paulo, 2016. Disponível em: http://www.sbem.com.br/enem2016/anais/pdf/7723_3560_ID.pdf. Acesso em: 12 de jan. 2020.

GUIMARÃES, Marcos Moraes; MATHIAS, Carmen Vieira. Ausência e necessidade de sinais adequados ao ensino de matemática para surdos. In: Encontro Nacional de Educação Matemática, anais do XII ENEM, São Paulo, 2016. Disponível em: http://www.sbembrasil.org.br/enem2016/anais/pdf/4820_2324_ID.pdf. Acesso em: 3 de jan. 2020.

KALEFF, Ana Maria Martensen Roland; ROSA, Fernanda Malinosky Coelho da; OLIVEIRA, Matheus Freitas. Um catálogo de materiais didáticos concretos e virtuais para um laboratório de ensino de matemática inclusiva. In: Encontro Nacional de Educação Matemática, anais do XII ENEM, São Paulo, 2016. Disponivel em: http://www.sbembrasil.org.br/enem2016/anais/pdf/5005_2699_ID.pdf. Acesso em: 13 de jan. 2020.

KENSKI, Vani Moreira. Educação e tecnologias: O novo ritmo da informação. Campinas: Papirus, 2007. 
LANUTTI, José Eduardo. Educação inclusiva. In: INSTITUTO IUNGO. Iungo convida :: Educação Inclusiva :: LEPED/UNICAMP. 2020. (1h11m14s). Disponível em: https://www.youtube.com/watch?v=Qs_trvCwKBQ\&t=2389s. Acesso em: 03 de jun. 2020.

LÉVY, Pierre. Cibercultura. Tradução de Carlos Irineu da Costa. São Paulo: Ed. 34, 1999.

LETWIN, Edith. Tecnologia educacional: Politica, histórias e propostas. Buenos Aires: Artmed, 1997.

LUZ, Adriana Augusta Benigno dos Santos; GÓES, Anderson Roges Teixeira. A expressão gráfica como tecnologia educacional na formação acadêmica docente. In: TERÇARIO, et al. Da internet para a sala de aula: tecnologia e comunicação no Brasil. Jundiaí: Paco editorial, 2016. p 41-50.

MANTOAN, Maria Tereza Eglér. Inclusão Escolar: O que é? Por quê? Como fazer? São Paulo, Moderna, 2003.

MANTOAN, Maria Tereza Eglér. Educação inclusiva. In: INSTITUTO IUNGO. Iungo convida :: Educação Inclusiva :: LEPED/UNICAMP. 2020. (1h11m14s). Disponível em: https://www.youtube.com/watch?v=Qs_trvCwKBQ\&t=2389s. Acesso em: 03 jun. 2020.

MORAES, Matheus Henrique Morato; DUTRA, Aline Gobbi. Audiovisual, Acessibilidade e as TICs a serviço da educação matemática: relatos do projeto "curtas matemáticos". In: Encontro Nacional de Educação Matemática, anais do XII ENEM, São Paulo, 2016. Disponível em: http://www.sbem.com.br/enem2016/anais/pdf/5716_3616_ID.pdf. Acesso em: 3 de jan. 2020.

MORAIS, Tula Maria Rocha; SILVA, Elisabete Leopoldina da.; FERNANDES, Solange Hassan Ahmad Ali. Investigando as possibilidades do Scrath para o ensino e a aprendizagem de conceitos matemáticos em cenários inclusivos. In: Encontro Nacional de Educação Matemática, anais do XIII ENEM, Cuiabá, 2019. Disponível em: https://www.sbemmatogrosso.com.br/eventos/index.php/enem/2019/paper/view/579/1078 Acesso em: 10 de fev. 2020.

ONU - Organização das Nações Unidas. Convenção Internacional do Direito das Pessoas com deficiência. 2006. Disponível em: https://www.un.org/development/desa/disabilities-es/convencion-sobre-los-derechos-delas-personas-con-discapacidad-2.html. Acesso em: 5 de abr. 2020.

ROCHA, Naiara Chierici; SCHLÜNZEN, Elisa.Tomoe Moriya. O ensino de matemática e inclusão escolar: a perspectiva de projetos e da abordagem CCS. In: Encontro Nacional de Educação Matemática, anais do XII ENEM, São Paulo, 2016. Disponível em: http://www.sbem.com.br/enem2016/anais/pdf/7384_3361_ID.pdf. Acesso em: 3 de jan. 2020.

SANCHO, Juana M. A tecnologia: um modo de transformar o mundo carregado de ambivalência. In Sancho, Juana M. (Org.). Para uma tecnologia educacional. Porto Alegre: Artmed, 1998. 
SANTOS, Carlos Eduardo Rocha dos; FERNANDES, Solange Hassam Ahmad Ali. O design universal na educação a distância: uma proposta de Curso de educação financeira. In: Encontro Nacional de Educação Matemática, anais do XII ENEM, São Paulo, 2016. Disponível em:

http://www.sbem.com.br/enem2016/anais/pdf/7891_3408_ID.pdf. Acesso em: 3 de jan. 2020.

SANTOS, Carlos Eduardo Rocha; FERNANDES, Solange Hassan Ahmad Ali. Cenários inclusivos para aprendizagem matemática: Utilização de aplicativos on-line. In: Encontro Nacional de Educação Matemática, anais do XIII ENEM, Cuiabá, 2019. Disponível em: https://www.sbemmatogrosso.com.br/eventos/index.php/enem/2019/paper/view/3128/111 4. Acesso em: 10 de fev. 2020.

SBEM - SOCIEDADE BRASILEIRA DE EDUCAÇÃO MATEMÁTICA. SBEM XIII: 2019. Pagina Inicial. Disponível em: http://www.sbemmatogrosso.com.br/xiienem/index.php./. Acesso em: 15 nov. 2019.

SBEM - SOCIEDADE BRASILEIRA DE EDUCAÇÃO MATEMÁTICA. GT13 - Diferença, Inclusão e Educação Matemática. 2020. Disponível em:

http://www.sbembrasil.org.br/sbembrasil/index.php/grupo-de-trabalho/gt/gt-13. Acesso em: $11 \mathrm{dez} .2020$.

UNESCO - ORGANIZAÇÃO DAS NAÇÕES UNIDAS PARA A EDUCAÇÃO, A CIÊNCIA E A CULTURA. Declaração de Salamanca e Enquadramento da Acção na área das necessidades educativas especiais. Salamanca - Espanha, 1994. Disponível em: http://portal.mec.gov.br/seesp/arquivos/pdf/salamanca.pdf. Acesso: 5 de abr. 2020.

ZANQUETA, Maria Emilia Melo Tamanini; NOGUEIRA, Cléia Maria Ignatius. TDAH, surdez e ensino de matemática: $O$ cálculo mental como estratégia didática. In: Encontro Nacional de Educação Matemática, anais do XII ENEM, São Paulo, 2016. Disponível em: http://www.sbembrasil.org.br/enem2016/anais/pdf/7326_3138_ID.pdf. Acesso em: 5 de jan. 2020.

ZERBATO, Ana Paula; MENDES, Enicéia Gonçalves. Desenho universal para a aprendizagem como estratégia de inclusão escolar. Revista Educação Unisinos, São Leopoldo RS, v22, n.2, 2018/abr-jun. Disponível em:

http://revistas.unisinos.br/index.php/educacao/article/view/edu.2018.222.04/60746207. Acesso em: 3 de jun. 2020. International (CC BY-NC 4.0) 